\title{
Comparing Causal Discovery Methods using Synthetic and Real Data
}

Christoph Käding and Jakob Runge

DLR German Aerospace Center Institute of Data Science, Jena, Germany

May 7, 2020
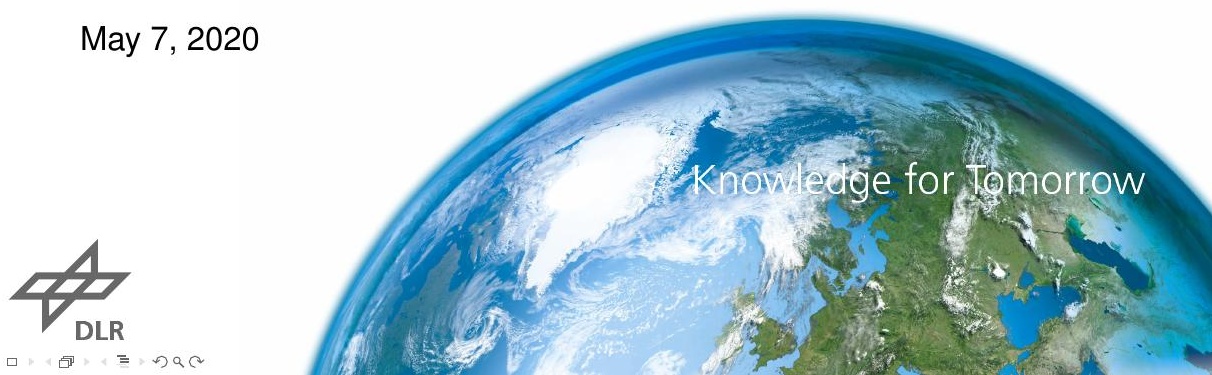
$>$ Unveiling causal structures, i.e., distinguishing cause from effect, from observational data plays a key role in climate science.

$>$ Various techniques are available to approach this while each comes with own assumptions about the data.

$\rightarrow$ restricted applicability

$>$ Goal: Evaluate and compare a number of state-of-the-art methods in a joint benchmark.

$>$ Synthetic data allows for controlling dataset conditions in detail.

$>$ Real data gives performance indicators for applications.

$>$ Disclaimer:

$>$ We concentrate on the case with two uni-variate variables.

$>$ This is an intermediate report of an ongoing study. 
Adaptive noise model (ANM) by [Hoyer et al., 2009]

$>$ decision according to regression residuals

Causal additive models (CAM) by [Bühlmann et al., 2014]

$>$ relies on fitting Gaussian processes

Concave penalized coordinate descent with reparametrization (CCDr) by [Aragam and Zhou, 2015]

$>$ utilizes a score-based structure learning approach

Conditional distribution similarity (CDS) by [Fonollosa, 2016]

$>$ decision according to the standard deviation of conditional distributions

Information geometric causal inference (IGCI) by [Daniusis et al., 2012]

$\checkmark$ relies on differential entropies 
Kernel conditional deviance for causal inference (KCDC) by [Mitrovic et al., 2018]

$\checkmark$ decision according to smaller deviance in the norms of conditional kernel mean embeddings

Linear non-Gaussian acyclic model (LiNGAM) by [Shimizu et al., 2006]

7 utilizes independent component analysis to resolve directions

Non-combinatorial optimization via trace exponential and augmented lagrangian for structure learning (noTEARS) by [Zheng et al., 2018]

$>$ reformulation of the problem as continuous optimization task

Randomized causation coefficient (RCC) by [Lopez-Paz et al., 2015]

$>$ transforms data into a feature representation and decides with the help of trained classifiers

Regression error based causal inference (RECI) by [Blöbaum et al., 2018]

$\checkmark$ decision according to least-squares errors of regressors fitted for both directions 


\section{Synthetic Data}

Synthetic data allows for constructing datasets with specific conditions. Therefore, we extend the functions used by [Mitrovic et al., 2018]:

$$
\begin{array}{ll}
\text { lin_a: } Y=X+10+\epsilon & \text { mul_a: } Y=\left(X^{3}+X\right) \cdot e^{\epsilon} \\
\text { lin_b: } Y=(X+10) \cdot \epsilon & \text { mul_b: } Y=\left(\sin (10 \cdot X)+e^{3 \cdot X}\right) \cdot e^{\epsilon} \\
\text { lin_c: } Y=(X+10)^{\epsilon} & \text { mul_c: } Y=\left(\log (X+10)+X^{6}\right) \cdot e^{\epsilon} \\
& \\
\text { add_a: } Y=X^{3}+X+\epsilon & \text { com_a: } Y=\left(\log (X+10)+X^{2}\right)^{\epsilon} \\
\text { add_b: } Y=\log (X+10)+X^{6}+\epsilon & \text { com_b: } Y=\log (X+10)+|X|^{2 \cdot|\epsilon|} \\
\text { add_c: } Y=\sin (10 \cdot X)+e^{3 \cdot X}+\epsilon & \text { com_c: } Y=\log \left(|X|^{7}+5\right)+X^{5}-\sin \left(X^{2} \cdot|\epsilon|\right)
\end{array}
$$

With:

$>$ cause $X \sim \mathcal{N}(0,1)$

$>$ noise $\epsilon \sim \mathcal{N}(0,1)$, or $\epsilon \sim \mathcal{U}(0,1)$, or $\epsilon \sim \operatorname{EXP}(1)$

$>100$ or 1000 data points per realization

$>100$ realizations each
Goal: Predict whether $X$ causes $Y$ or $Y$ causes $X$.

Please note: Due to implementation details of the methods, we actually deal with a three class problem $(X \rightarrow Y, X \leftarrow Y$, and $X \Perp Y$ ) while predicting the third class is always wrong. 
Correct estimates overall: $48.361 \%(1741 / 3600$, bootstrap err. $0.815 \%)$

$\begin{array}{ll}\text { Correct predictions for } & \text { individual setups: } \\ \text { lin_a + normal } & 54.000 \%(54 / 100) \\ \text { lin_a + uniform } & 81.000 \%(81 / 100) \\ \text { lin_a + exponential } & 90.000 \%(90 / 100) \\ \text { lin_b + normal } & 47.000 \%(47 / 100) \\ \text { lin_b + uniform } & 28.000 \%(28 / 100) \\ \text { lin_b + exponential } & 17.000 \%(17 / 100) \\ \text { lin_c + normal } & 1.000 \%(1 / 100) \\ \text { lin_c + uniform } & 8.000 \%(8 / 100) \\ \text { lin_c + exponential } & 0.000 \%(0 / 100) \\ \text { add_a + normal } & 100.000 \%(100 / 100) \\ \text { add_a + uniform } & 100.000 \%(100 / 100) \\ \text { add_a + exponential } & 98.000 \%(98 / 100) \\ \text { add_b + normal } & 100.000 \%(100 / 100) \\ \text { add_b + uniform } & 99.000 \%(99 / 100) \\ \text { add_b + exponential } & 97.000 \%(97 / 100) \\ \text { add_c + normal } & 100.000 \%(100 / 100) \\ \text { add_c + uniform } & 100.000 \%(100 / 100) \\ \text { add_c + exponential } & 100.000 \%(100 / 100) \\ \text { mul_a + normal } & 1.000 \%(1 / 100) \\ \text { mul_a + uniform } & 0.000 \%(0 / 100) \\ \text { mul_a + exponential } & 10.000 \%(10 / 100) \\ \text { mul_b + normal } & 0.000 \%(0 / 100) \\ \text { mul_b + uniform } & 4.000 \%(4 / 100) \\ \text { mul_b + exponential } & 5.000 \%(5 / 100) \\ \text { mul_c + normal } & 10.000 \%(10 / 100) \\ \text { mul_c + uniform } & 35.000 \%(35 / 100) \\ \text { mul_c + exponential } & 8.000 \%(8 / 100) \\ & \end{array}$

com_a + normal

com_a + uniform

com_a + exponential

com_b + normal

com_b + uniform

com_b + exponential

com_c + normal

com_c + uniform

com_c + exponential

Correct predictions averaged for function type:

$\begin{array}{ll}\text { lin_a } & 75.000 \%(225 / 300) \\ \text { lin_b } & 30.667 \%(92 / 300) \\ \text { lin_c } & 3.000 \%(9 / 300) \\ \text { add_a } & 99.333 \%(298 / 300) \\ \text { add_b } & 98.667 \%(296 / 300) \\ \text { add_c } & 100.000 \%(300 / 300) \\ \text { mul_a } & 3.667 \%(11 / 300) \\ \text { mul_b } & 3.000 \%(9 / 300) \\ \text { mul_c } & 17.667 \%(53 / 300) \\ \text { com_a } & 9.667 \%(29 / 300) \\ \text { com_b } & 98.000 \%(294 / 300) \\ \text { com_c } & 41.667 \%(125 / 300)\end{array}$

Correct predictions averaged for noise type:

normal

uniform

$46.417 \%$ (557 / 1200)

exponential

$51.417 \%(617 / 1200)$

$47.250 \%(567 / 1200)$ 


\section{ANM Results for Synthetic (1000)}

Correct estimates overall: $48.139 \%$ (1733 / 3600, bootstrap err. $0.836 \%)$

$\begin{array}{ll}\text { Correct predictions for individual setups: } \\ \text { lin_a + normal } & 48.000 \%(48 / 100) \\ \text { lin_a + uniform } & 99.000 \%(99 / 100) \\ \text { lin_a + exponential } & 100.000 \%(100 / 100) \\ \text { lin_b + normal } & 30.000 \%(30 / 100) \\ \text { lin_b + uniform } & 30.000 \%(30 / 100) \\ \text { lin_b + exponential } & 19.000 \%(19 / 100) \\ \text { lin_c + normal } & 0.000 \%(0 / 100) \\ \text { lin_c + uniform } & 10.000 \%(10 / 100) \\ \text { lin_c + exponential } & 0.000 \%(0 / 100) \\ \text { add_a + normal } & 100.000 \%(100 / 100) \\ \text { add_a + uniform } & 100.000 \%(100 / 100) \\ \text { add_a + exponential } & 100.000 \%(100 / 100) \\ \text { add_b + normal } & 100.000 \%(100 / 100) \\ \text { add_b + uniform } & 100.000 \%(100 / 100) \\ \text { add_b + exponential } & 100.000 \%(100 / 100) \\ \text { add_c + normal } & 100.000 \%(100 / 100) \\ \text { add_c + uniform } & 100.000 \%(100 / 100) \\ \text { add_c + exponential } & 100.000 \%(100 / 100) \\ \text { mul_a + normal } & 0.000 \%(0 / 100) \\ \text { mul_a + uniform } & 0.000 \%(0 / 100) \\ \text { mul_a + exponential } & 27.000 \%(27 / 100) \\ \text { mul_b + normal } & 8.000 \%(8 / 100) \\ \text { mul_b + uniform } & 29.000 \%(29 / 100) \\ \text { mul_b + exponential } & 22.000 \%(22 / 100) \\ \text { mul_c + normal } & 15.000 \%(15 / 100) \\ \text { mul_c + uniform } & 76.000 \%(76 / 100) \\ \text { mul_c + exponential } & 14.000 \%(14 / 100)\end{array}$

com_a + normal

com_a + uniform

com_a + exponential

com_b + normal

com_b + uniform

com_b + exponential

com_c + normal

com_c + uniform

com_c + exponential

Correct predictions averaged for function type:

$\begin{array}{ll}\text { lin_a } & 82.333 \%(247 / 300) \\ \text { lin_b } & 26.333 \%(79 / 300) \\ \text { lin_c } & 3.333 \%(10 / 300) \\ \text { add_a } & 100.000 \%(300 / 300) \\ \text { add_b } & 100.000 \%(300 / 300) \\ \text { add_c } & 100.000 \%(300 / 300) \\ \text { mul_a } & 9.000 \%(27 / 300) \\ \text { mul_b } & 19.667 \%(59 / 300) \\ \text { mul_c } & 35.000 \%(105 / 300) \\ \text { com_a } & 0.667 \%(2 / 300) \\ \text { com_b } & 99.333 \%(298 / 300) \\ \text { com_c } & 2.000 \%(6 / 300)\end{array}$

Correct predictions averaged for noise type:

normal

$42.000 \%$ (504 / 1200)

uniform

$53.833 \%(646 / 1200)$

exponential

$48.583 \%$ (583 / 1200) 
Correct estimates overall: $70.389 \%$ (2534 / 3600, bootstrap err. $0.749 \%$ )

$\begin{array}{ll}\text { Correct predictions for individual setups: } \\ \text { lin_a + normal } & 45.000 \%(45 / 100) \\ \text { lin_a + uniform } & 53.000 \%(53 / 100) \\ \text { lin_a + exponential } & 4.000 \%(4 / 100) \\ \text { lin_b + normal } & 30.000 \%(30 / 100) \\ \text { lin_b + uniform } & 15.000 \%(15 / 100) \\ \text { lin_b + exponential } & 52.000 \%(52 / 100) \\ \text { lin_c + normal } & 42.000 \%(42 / 100) \\ \text { lin_c + uniform } & 48.000 \%(48 / 100) \\ \text { lin_c + exponential } & 42.000 \%(42 / 100) \\ \text { add_a + normal } & 100.000 \%(100 / 100) \\ \text { add_a + uniform } & 100.000 \%(100 / 100) \\ \text { add_a + exponential } & 100.000 \%(100 / 100) \\ \text { add_b + normal } & 100.000 \%(100 / 100) \\ \text { add_b + uniform } & 100.000 \%(100 / 100) \\ \text { add_b + exponential } & 100.000 \%(100 / 100) \\ \text { add_c + normal } & 100.000 \%(100 / 100) \\ \text { add_c + uniform } & 100.000 \%(100 / 100) \\ \text { add_c + exponential } & 100.000 \%(100 / 100) \\ \text { mul_a + normal } & 6.000 \%(6 / 100) \\ \text { mul_a + uniform } & 6.000 \%(6 / 100) \\ \text { mul_a + exponential } & 1.000 \%(1 / 100) \\ \text { mul_b + normal } & 69.000 \%(69 / 100) \\ \text { mul_b + uniform } & 96.000 \%(96 / 100) \\ \text { mul_b + exponential } & 60.000 \%(60 / 100) \\ \text { mul_c + normal } & 100.000 \%(100 / 100) \\ \text { mul_c + uniform } & 100.000 \%(100 / 100) \\ \text { mul_c + exponential } & 87.000 \%(87 / 100)\end{array}$

com_a + normal

com_a + uniform

com_a + exponential

com_b + normal

com_b + uniform

com_b + exponential

com_c + normal

com_c + uniform

com_c + exponential

$69.000 \%(69 / 100)$

$59.000 \%(59 / 100)$

$64.000 \%(64 / 100)$

$97.000 \%(97 / 100)$

$100.000 \%(100 / 100)$

$89.000 \%(89 / 100)$

$100.000 \%(100 / 100)$

$100.000 \%(100 / 100)$

$100.000 \%(100 / 100)$

\section{Correct predictions averaged for function type:}

$\begin{array}{ll}\text { lin_a } & 34.000 \%(102 / 300) \\ \text { lin_b } & 32.333 \%(97 / 300) \\ \text { lin_c } & 44.000 \%(132 / 300) \\ \text { add_a } & 100.000 \%(300 / 300) \\ \text { add_b } & 100.000 \%(300 / 300) \\ \text { add_c } & 100.000 \%(300 / 300) \\ \text { mul_a } & 4.333 \%(13 / 300) \\ \text { mul_b } & 75.000 \%(225 / 300) \\ \text { mul_c } & 95.667 \%(287 / 300) \\ \text { com_a } & 64.000 \%(192 / 300) \\ \text { com_b } & 95.333 \%(286 / 300) \\ \text { com_c } & 100.000 \%(300 / 300)\end{array}$

Correct predictions averaged for noise type:

normal: $71.500 \%(858 / 1200)$

uniform: $73.083 \%(877 / 1200)$

exponential: $66.583 \%(799 / 1200)$ 
Correct estimates overall: $69.306 \%$ (2495 / 3600, bootstrap err. $0.769 \%)$

$\begin{array}{ll}\text { Correct predictions for individual setups: } \\ \text { lin_a + normal } & 53.000 \%(53 / 100) \\ \text { lin_a + uniform } & 43.000 \%(43 / 100) \\ \text { lin_a + exponential } & 0.000 \%(0 / 100) \\ \text { lin_b + normal } & 0.000 \%(0 / 100) \\ \text { lin_b + uniform } & 0.000 \%(0 / 100) \\ \text { lin_b + exponential } & 43.000 \%(43 / 100) \\ \text { lin_c + normal } & 40.000 \%(40 / 100) \\ \text { lin_c + uniform } & 6.000 \%(6 / 100) \\ \text { lin_c + exponential } & 40.000 \%(40 / 100) \\ \text { add_a + normal } & 100.000 \%(100 / 100) \\ \text { add_a + uniform } & 100.000 \%(100 / 100) \\ \text { add_a + exponential } & 100.000 \%(100 / 100) \\ \text { add_b + normal } & 100.000 \%(100 / 100) \\ \text { add_b + uniform } & 100.000 \%(100 / 100) \\ \text { add_b + exponential } & 100.000 \%(100 / 100) \\ \text { add_c + normal } & 100.000 \%(100 / 100) \\ \text { add_c + uniform } & 100.000 \%(100 / 100) \\ \text { add_c + exponential } & 100.000 \%(100 / 100) \\ \text { mul_a + normal } & 0.000 \%(0 / 100) \\ \text { mul_a + uniform } & 0.000 \%(0 / 100) \\ \text { mul_a + exponential } & 0.000 \%(0 / 100) \\ \text { mul_b + normal } & 85.000 \%(85 / 100) \\ \text { mul_b + uniform } & 100.000 \%(100 / 100) \\ \text { mul_b + exponential } & 41.000 \%(41 / 100) \\ \text { mul_c + normal } & 100.000 \%(100 / 100) \\ \text { mul_c + uniform } & 100.000 \%(100 / 100) \\ \text { mul_c + exponential } & 88.000 \%(88 / 100) \\ & \end{array}$

com_a + normal

com_a + uniform

com_a + exponential

com_b + normal

com_b + uniform

com_b + exponential

com_c + normal

com_c + uniform

com_c + exponential

$97.000 \%(97 / 100)$

$95.000 \%(95 / 100)$

$74.000 \%(74 / 100)$

$99.000 \%(99 / 100)$

$100.000 \%(100 / 100)$

$91.000 \%(91 / 100)$

$100.000 \%(100 / 100)$

$100.000 \%(100 / 100)$

$100.000 \%(100 / 100)$

\section{Correct predictions averaged for function type:}

$\begin{array}{ll}\text { lin_a } & 32.000 \%(96 / 300) \\ \text { lin_b } & 14.333 \%(43 / 300) \\ \text { lin_c } & 28.667 \%(86 / 300) \\ \text { add_a } & 100.000 \%(300 / 300) \\ \text { add_b } & 100.000 \%(300 / 300) \\ \text { add_c } & 100.000 \%(300 / 300) \\ \text { mul_a } & 0.000 \%(0 / 300) \\ \text { mul_b } & 75.333 \%(226 / 300) \\ \text { mul_c } & 96.000 \%(288 / 300) \\ \text { com_a } & 88.667 \%(266 / 300) \\ \text { com_b } & 96.667 \%(290 / 300) \\ \text { com_c } & 100.000 \%(300 / 300)\end{array}$

Correct predictions averaged for noise type:

normal: $72.833 \%(874 / 1200)$

uniform: $70.333 \%(844 / 1200)$

exponential: $64.750 \%(777 / 1200)$ 


\section{CCDr Results for Synthetic (100)}

Correct estimates overall: $50.028 \%$ (1801 / 3600, bootstrap err. $0.832 \%)$

$\begin{array}{ll}\text { Correct predictions for } & \text { individual setups: } \\ \text { lin_a + normal } & 55.000 \%(55 / 100) \\ \text { lin_a + uniform } & 51.000 \%(51 / 100) \\ \text { lin_a + exponential } & 50.000 \%(50 / 100) \\ \text { lin_b + normal } & 46.000 \%(46 / 100) \\ \text { lin_b + uniform } & 50.000 \%(50 / 100) \\ \text { lin_b + exponential } & 52.000 \%(52 / 100) \\ \text { lin_c + normal } & 53.000 \%(53 / 100) \\ \text { lin_c + uniform } & 47.000 \%(47 / 100) \\ \text { lin_c + exponential } & 47.000 \%(47 / 100) \\ \text { add_a + normal } & 57.000 \%(57 / 100) \\ \text { add_a + uniform } & 56.000 \%(56 / 100) \\ \text { add_a + exponential } & 50.000 \%(50 / 100) \\ \text { add_b + normal } & 43.000 \%(43 / 100) \\ \text { add_b + uniform } & 48.000 \%(48 / 100) \\ \text { add_b + exponential } & 40.000 \%(40 / 100) \\ \text { add_c + normal } & 54.000 \%(54 / 100) \\ \text { add_c + uniform } & 48.000 \%(48 / 100) \\ \text { add_c + exponential } & 51.000 \%(51 / 100) \\ \text { mul_a + normal } & 50.000 \%(50 / 100) \\ \text { mul_a + uniform } & 53.000 \%(53 / 100) \\ \text { mul_a + exponential } & 46.000 \%(46 / 100) \\ \text { mul_b + normal } & 60.000 \%(60 / 100) \\ \text { mul_b + uniform } & 50.000 \%(50 / 100) \\ \text { mul_b + exponential } & 60.000 \%(60 / 100) \\ \text { mul_c + normal } & 50.000 \%(50 / 100) \\ \text { mul_c + uniform } & 43.000 \%(43 / 100) \\ \text { mul_c + exponential } & 49.000 \%(49 / 100)\end{array}$

com_a + normal

com_a + uniform

com_a + exponential

com_b + normal

com_b + uniform

com_b + exponential

com_c + normal

com $c+$ uniform

com $c$ + exponential

Correct predictions averaged for function type:

$\begin{array}{ll}\text { lin_a } & 52.000 \%(156 / 300) \\ \text { lin_b } & 49.333 \%(148 / 300) \\ \text { lin_c } & 49.000 \%(147 / 300) \\ \text { add_a } & 54.333 \%(163 / 300) \\ \text { add_b } & 43.667 \%(131 / 300) \\ \text { add_c } & 51.000 \%(153 / 300) \\ \text { mul_a } & 49.667 \%(149 / 300) \\ \text { mul_b } & 56.667 \%(170 / 300) \\ \text { mul_c } & 47.333 \%(142 / 300) \\ \text { com_a } & 50.000 \%(150 / 300) \\ \text { com_b } & 48.000 \%(144 / 300) \\ \text { com_c } & 49.333 \%(148 / 300)\end{array}$

Correct predictions averaged for noise type:

normal: $51.667 \%(620 / 1200)$

uniform: $48.583 \%(583 / 1200)$

exponential: $49.833 \%(598 / 1200)$ 


\section{CCDr Results for Synthetic (1000)}

Correct estimates overall: $47.028 \%$ (1693 / 3600, bootstrap err. $0.806 \%$ )

$\begin{array}{ll}\text { Correct predictions for } & \text { individual setups: } \\ \text { lin_a + normal } & 53.000 \%(53 / 100) \\ \text { lin_a + uniform } & 59.000 \%(59 / 100) \\ \text { lin_a + exponential } & 51.000 \%(51 / 100) \\ \text { lin_b + normal } & 36.000 \%(36 / 100) \\ \text { lin_b + uniform } & 44.000 \%(44 / 100) \\ \text { lin_b + exponential } & 47.000 \%(47 / 100) \\ \text { lin_c + normal } & 32.000 \%(32 / 100) \\ \text { lin_c + uniform } & 50.000 \%(50 / 100) \\ \text { lin_c + exponential } & 39.000 \%(39 / 100) \\ \text { add_a + normal } & 55.000 \%(55 / 100) \\ \text { add_a + uniform } & 49.000 \%(49 / 100) \\ \text { add_a + exponential } & 52.000 \%(52 / 100) \\ \text { add_b + normal } & 50.000 \%(50 / 100) \\ \text { add_b + uniform } & 44.000 \%(44 / 100) \\ \text { add_b + exponential } & 38.000 \%(38 / 100) \\ \text { add_c + normal } & 47.000 \%(47 / 100) \\ \text { add_c + uniform } & 47.000 \%(47 / 100) \\ \text { add_c + exponential } & 58.000 \%(58 / 100) \\ \text { mul_a + normal } & 48.000 \%(48 / 100) \\ \text { mul_a + uniform } & 51.000 \%(51 / 100) \\ \text { mul_a + exponential } & 46.000 \%(46 / 100) \\ \text { mul_b + normal } & 50.000 \%(50 / 100) \\ \text { mul_b + uniform } & 45.000 \%(45 / 100) \\ \text { mul_b + exponential } & 45.000 \%(45 / 100) \\ \text { mul_c + normal } & 55.000 \%(55 / 100) \\ \text { mul_c + uniform } & 44.000 \%(44 / 100) \\ \text { mul_c + exponential } & 42.000 \%(42 / 100)\end{array}$

com_a + normal

com_a + uniform

com_a + exponential

com_b + normal

com_b + uniform

com_b + exponential

com_c + normal

com_c + uniform

com_c + exponential

$39.000 \%(39 / 100)$

$41.000 \%(41 / 100)$

$51.000 \%(51 / 100)$

$35.000 \%(35 / 100)$

$47.000 \%(47 / 100)$

$58.000 \%(58 / 100)$

$54.000 \%(54 / 100)$

$44.000 \%(44 / 100)$

$47.000 \%(47 / 100)$

Correct predictions averaged for function type:

$\begin{array}{ll}\text { lin_a } & 54.333 \%(163 / 300) \\ \text { lin_b } & 42.333 \%(127 / 300) \\ \text { lin_c } & 40.333 \%(121 / 300) \\ \text { add_a } & 52.000 \%(156 / 300) \\ \text { add_b } & 44.000 \%(132 / 300) \\ \text { add_c } & 50.667 \%(152 / 300) \\ \text { mul_a } & 48.333 \%(145 / 300) \\ \text { mul_b } & 46.667 \%(140 / 300) \\ \text { mul_c } & 47.000 \%(141 / 300) \\ \text { com_a } & 43.667 \%(131 / 300) \\ \text { com_b } & 46.667 \%(140 / 300) \\ \text { com_c } & 48.333 \%(145 / 300)\end{array}$

Correct predictions averaged for noise type:

normal: $46.167 \%$ (554 / 1200)

uniform: $47.083 \%(565 / 1200)$

exponential: $47.833 \%(574 / 1200)$ 
Correct estimates overall: $53.472 \%$ (1925 / 3600, bootstrap err. $0.832 \%)$

$\begin{array}{ll}\text { Correct predictions for } & \text { individual setups: } \\ \text { lin_a + normal } & 53.000 \%(53 / 100) \\ \text { lin_a + uniform } & 67.000 \%(67 / 100) \\ \text { lin_a + exponential } & 55.000 \%(55 / 100) \\ \text { lin_b + normal } & 46.000 \%(46 / 100) \\ \text { lin_b + uniform } & 51.000 \%(51 / 100) \\ \text { lin_b + exponential } & 22.000 \%(22 / 100) \\ \text { lin_c + normal } & 7.000 \%(7 / 100) \\ \text { lin_c + uniform } & 6.000 \%(6 / 100) \\ \text { lin_c + exponential } & 3.000 \%(3 / 100) \\ \text { add_a + normal } & 63.000 \%(63 / 100) \\ \text { add_a + uniform } & 78.000 \%(78 / 100) \\ \text { add_a + exponential } & 50.000 \%(50 / 100) \\ \text { add_b + normal } & 85.000 \%(85 / 100) \\ \text { add_b + uniform } & 76.000 \%(76 / 100) \\ \text { add_b + exponential } & 86.000 \%(86 / 100) \\ \text { add_c + normal } & 68.000 \%(68 / 100) \\ \text { add_c + uniform } & 68.000 \%(68 / 100) \\ \text { add_c + exponential } & 81.000 \%(81 / 100) \\ \text { mul_a + normal } & 33.000 \%(33 / 100) \\ \text { mul_a + uniform } & 43.000 \%(43 / 100) \\ \text { mul_a + exponential } & 37.000 \%(37 / 100) \\ \text { mul_b + normal } & 62.000 \%(62 / 100) \\ \text { mul_b + uniform } & 74.000 \%(74 / 100) \\ \text { mul_b + exponential } & 55.000 \%(55 / 100) \\ \text { mul_c + normal } & 91.000 \%(91 / 100) \\ \text { mul_c + uniform } & 90.000 \%(90 / 100) \\ \text { mul_c + exponential } & 65.000 \%(65 / 100)\end{array}$

com_a + normal

com_a + uniform

com_a + exponential

com_b + normal

com_b + uniform

com_b + exponential

com_c + normal

com_c + uniform

com_c + exponential

$29.000 \%(29 / 100)$

$28.000 \%(28 / 100)$

$41.000 \%(41 / 100)$

$98.000 \%(98 / 100)$

$97.000 \%(97 / 100)$

$90.000 \%(90 / 100)$

$7.000 \%(7 / 100)$

$6.000 \%(6 / 100)$

$14.000 \%(14 / 100)$

Correct predictions averaged for function type:

$\begin{array}{ll}\text { lin_a } & 58.333 \%(175 / 300) \\ \text { lin_b } & 39.667 \%(119 / 300) \\ \text { lin_c } & 5.333 \%(16 / 300) \\ \text { add_a } & 63.667 \%(191 / 300) \\ \text { add_b } & 82.333 \%(247 / 300) \\ \text { add_c } & 72.333 \%(217 / 300) \\ \text { mul_a } & 37.667 \%(113 / 300) \\ \text { mul_b } & 63.667 \%(191 / 300) \\ \text { mul_c } & 82.000 \%(246 / 300) \\ \text { com_a } & 32.667 \%(98 / 300) \\ \text { com_b } & 95.000 \%(285 / 300) \\ \text { com_c } & 9.000 \%(27 / 300)\end{array}$

Correct predictions averaged for noise type:

normal: $53.500 \%(642 / 1200)$

uniform: $57.000 \%(684 / 1200)$

exponential: $49.917 \%(599 / 1200)$ 
Correct estimates overall: $51.139 \%$ (1841/3600, bootstrap err. $0.817 \%)$

$\begin{array}{ll}\text { Correct predictions for } & \text { individual setups: } \\ \text { lin_a + normal } & 47.000 \%(47 / 100) \\ \text { lin_a + uniform } & 99.000 \%(99 / 100) \\ \text { lin_a + exponential } & 99.000 \%(99 / 100) \\ \text { lin_b + normal } & 43.000 \%(43 / 100) \\ \text { lin_b + uniform } & 25.000 \%(25 / 100) \\ \text { lin_b + exponential } & 6.000 \%(6 / 100) \\ \text { lin_c + normal } & 12.000 \%(12 / 100) \\ \text { lin_c + uniform } & 1.000 \%(1 / 100) \\ \text { lin_c + exponential } & 10.000 \%(10 / 100) \\ \text { add_a + normal } & 100.000 \%(100 / 100) \\ \text { add_a + uniform } & 60.000 \%(60 / 100) \\ \text { add_a + exponential } & 95.000 \%(95 / 100) \\ \text { add_b + normal } & 100.000 \%(100 / 100) \\ \text { add_b + uniform } & 100.000 \%(100 / 100) \\ \text { add_b + exponential } & 98.000 \%(98 / 100) \\ \text { add_c + normal } & 91.000 \%(91 / 100) \\ \text { add_c + uniform } & 97.000 \%(97 / 100) \\ \text { add_c + exponential } & 91.000 \%(91 / 100) \\ \text { mul_a + normal } & 0.000 \%(0 / 100) \\ \text { mul_a + uniform } & 0.000 \%(0 / 100) \\ \text { mul_a + exponential } & 5.000 \%(5 / 100) \\ \text { mul_b + normal } & 31.000 \%(31 / 100) \\ \text { mul_b + uniform } & 58.000 \%(58 / 100) \\ \text { mul_b + exponential } & 21.000 \%(21 / 100) \\ \text { mul_c + normal } & 33.000 \%(33 / 100) \\ \text { mul_c + uniform } & 77.000 \%(77 / 100) \\ \text { mul_c + exponential } & 29.000 \%(29 / 100) \\ & \end{array}$

com_a + normal

com_a + uniform

com_a + exponential

com_b + normal

com_b + uniform

com_b + exponential

com_c + normal

com_c + uniform

com_c + exponential

Correct predictions averaged for function type:

$\begin{array}{ll}\text { lin_a } & 81.667 \%(245 / 300) \\ \text { lin_b } & 24.667 \%(74 / 300) \\ \text { lin_c } & 7.667 \%(23 / 300) \\ \text { add_a } & 85.000 \%(255 / 300) \\ \text { add_b } & 99.333 \%(298 / 300) \\ \text { add_c } & 93.000 \%(279 / 300) \\ \text { mul_a } & 1.667 \%(5 / 300) \\ \text { mul_b } & 36.667 \%(110 / 300) \\ \text { mul_c } & 46.333 \%(139 / 300) \\ \text { com_a } & 27.000 \%(81 / 300) \\ \text { com_b } & 49.333 \%(148 / 300) \\ \text { com_c } & 61.333 \%(184 / 300)\end{array}$

Correct predictions averaged for noise type:

normal: $46.500 \%(558 / 1200)$

uniform: $62.000 \%(744 / 1200)$

exponential: $44.917 \%(539 / 1200)$ 
Correct estimates overall: $93.389 \%$ (3362 / 3600, bootstrap err. $0.415 \%$ )

$\begin{array}{ll}\text { Correct predictions for } & \text { individual setups: } \\ \text { lin_a + normal } & 42.000 \%(42 / 100) \\ \text { lin_a + uniform } & 54.000 \%(54 / 100) \\ \text { lin_a + exponential } & 61.000 \%(61 / 100) \\ \text { lin_b + normal } & 40.000 \%(40 / 100) \\ \text { lin_b + uniform } & 79.000 \%(79 / 100) \\ \text { lin_b + exponential } & 100.000 \%(100 / 100) \\ \text { lin_c + normal } & 100.000 \%(100 / 100) \\ \text { lin_c + uniform } & 100.000 \%(100 / 100) \\ \text { lin_c + exponential } & 100.000 \%(100 / 100) \\ \text { add_a + normal } & 96.000 \%(96 / 100) \\ \text { add_a + uniform } & 98.000 \%(98 / 100) \\ \text { add_a + exponential } & 99.000 \%(99 / 100) \\ \text { add_b + normal } & 100.000 \%(100 / 100) \\ \text { add_b + uniform } & 100.000 \%(100 / 100) \\ \text { add_b + exponential } & 100.000 \%(100 / 100) \\ \text { add_c + normal } & 100.000 \%(100 / 100) \\ \text { add_c + uniform } & 100.000 \%(100 / 100) \\ \text { add_c + exponential } & 100.000 \%(100 / 100) \\ \text { mul_a + normal } & 100.000 \%(100 / 100) \\ \text { mul_a + uniform } & 99.000 \%(99 / 100) \\ \text { mul_a + exponential } & 100.000 \%(100 / 100) \\ \text { mul_b + normal } & 100.000 \%(100 / 100) \\ \text { mul_b + uniform } & 100.000 \%(100 / 100) \\ \text { mul_b + exponential } & 100.000 \%(100 / 100) \\ \text { mul_c + normal } & 100.000 \%(100 / 100) \\ \text { mul_c + uniform } & 100.000 \%(100 / 100) \\ \text { mul_c + exponential } & 100.000 \%(100 / 100)\end{array}$

com_a + normal

com_a + uniform

com_a + exponential

com_b + normal

com_b + uniform

com_b + exponential

com_c + normal

com_c + uniform

com_c + exponential
$100.000 \%(100 / 100)$ $99.000 \%(99 / 100)$ $100.000 \%(100 / 100)$ $100.000 \%(100 / 100)$ $95.000 \%(95 / 100)$ $100.000 \%(100 / 100)$ $100.000 \%(100 / 100)$ $100.000 \%(100 / 100)$ $100.000 \%(100 / 100)$

Correct predictions averaged for function type:

$\begin{array}{ll}\text { lin_a } & 52.333 \%(157 / 300) \\ \text { lin_b } & 73.000 \%(219 / 300) \\ \text { lin_c } & 100.000 \%(300 / 300) \\ \text { add_a } & 97.667 \%(293 / 300) \\ \text { add_b } & 100.000 \%(300 / 300) \\ \text { add_c } & 100.000 \%(300 / 300) \\ \text { mul_a } & 99.667 \%(299 / 300) \\ \text { mul_b } & 100.000 \%(300 / 300) \\ \text { mul_c } & 100.000 \%(300 / 300) \\ \text { com_a } & 99.667 \%(299 / 300) \\ \text { com_b } & 98.333 \%(295 / 300) \\ \text { com_c } & 100.000 \%(300 / 300)\end{array}$

Correct predictions averaged for noise type: normal: $89.833 \%(1078 / 1200)$ uniform: $93.667 \%(1124 / 1200)$ exponential: $96.667 \%(1160 / 1200)$ 


\section{IGCI Results for Synthetic (1000)}

Correct estimates overall: $95.306 \%$ (3431 / 3600, bootstrap err. $0.352 \%$ )

$\begin{array}{ll}\text { Correct predictions for individual setups: } \\ \text { lin_a + normal } & 61.000 \%(61 / 100) \\ \text { lin_a + uniform } & 50.000 \%(50 / 100) \\ \text { lin_a + exponential } & 74.000 \%(74 / 100) \\ \text { lin_b + normal } & 46.000 \%(46 / 100) \\ \text { lin_b + uniform } & 100.000 \%(100 / 100) \\ \text { lin_b + exponential } & 100.000 \%(100 / 100) \\ \text { lin_c + normal } & 100.000 \%(100 / 100) \\ \text { lin_c + uniform } & 100.000 \%(100 / 100) \\ \text { lin_c + exponential } & 100.000 \%(100 / 100) \\ \text { add_a + normal } & 100.000 \%(100 / 100) \\ \text { add_a + uniform } & 100.000 \%(100 / 100) \\ \text { add_a + exponential } & 100.000 \%(100 / 100) \\ \text { add_b + normal } & 100.000 \%(100 / 100) \\ \text { add_b + uniform } & 100.000 \%(100 / 100) \\ \text { add_b + exponential } & 100.000 \%(100 / 100) \\ \text { add_c + normal } & 100.000 \%(100 / 100) \\ \text { add_c + uniform } & 100.000 \%(100 / 100) \\ \text { add_c + exponential } & 100.000 \%(100 / 100) \\ \text { mul_a + normal } & 100.000 \%(100 / 100) \\ \text { mul_a + uniform } & 100.000 \%(100 / 100) \\ \text { mul_a + exponential } & 100.000 \%(100 / 100) \\ \text { mul_b + normal } & 100.000 \%(100 / 100) \\ \text { mul_b + uniform } & 100.000 \%(100 / 100) \\ \text { mul_b + exponential } & 100.000 \%(100 / 100) \\ \text { mul_c + normal } & 100.000 \%(100 / 100) \\ \text { mul_c + uniform } & 100.000 \%(100 / 100) \\ \text { mul_c + exponential } & 100.000 \%(100 / 100)\end{array}$

com_a + normal

com_a + uniform

com_a + exponential

com_b + normal

com_b + uniform

com_b + exponential

com_c + normal

com_c + uniform

com $\mathrm{c}+$ exponential
$100.000 \%(100 / 100)$ $100.000 \%(100 / 100)$ $100.000 \%(100 / 100)$ $100.000 \%(100 / 100)$ $100.000 \%(100 / 100)$ $100.000 \%(100 / 100)$ $100.000 \%(100 / 100)$ $100.000 \%(100 / 100)$ $100.000 \%(100 / 100)$

\section{Correct predictions averaged for function type:}

$\begin{array}{ll}\text { lin_a } & 61.667 \%(185 / 300) \\ \text { lin_b } & 82.000 \%(246 / 300) \\ \text { lin_c } & 100.000 \%(300 / 300) \\ \text { add_a } & 100.000 \%(300 / 300) \\ \text { add_b } & 100.000 \%(300 / 300) \\ \text { add_c } & 100.000 \%(300 / 300) \\ \text { mul_a } & 100.000 \%(300 / 300) \\ \text { mul_b } & 100.000 \%(300 / 300) \\ \text { mul_c } & 100.000 \%(300 / 300) \\ \text { com_a } & 100.000 \%(300 / 300) \\ \text { com_b } & 100.000 \%(300 / 300) \\ \text { com_c } & 100.000 \%(300 / 300)\end{array}$

Correct predictions averaged for noise type: normal: $92.250 \%(1107 / 1200)$ uniform: $95.833 \%(1150 / 1200)$ exponential: $97.833 \%(1174$ / 1200) 


\section{KCDC Results for Synthetic (100)}

Correct estimates overall: overall: $9.278 \%$ (334 / 3600, bootstrap err. $0.478 \%)$

$\begin{array}{ll}\text { Correct predictions for individual setups: } \\ \text { lin_a + normal } & 0.000 \%(0 / 100) \\ \text { lin_a + uniform } & 1.000 \%(1 / 100) \\ \text { lin_a + exponential } & 0.000 \%(0 / 100) \\ \text { lin_b + normal } & 27.000 \%(27 / 100) \\ \text { lin_b + uniform } & 0.000 \%(0 / 100) \\ \text { lin_b + exponential } & 1.000 \%(1 / 100) \\ \text { lin_c + normal } & 0.000 \%(0 / 100) \\ \text { lin_c + uniform } & 0.000 \%(0 / 100) \\ \text { lin_c + exponential } & 0.000 \%(0 / 100) \\ \text { add_a + normal } & 0.000 \%(0 / 100) \\ \text { add_a + uniform } & 0.000 \%(0 / 100) \\ \text { add_a + exponential } & 0.000 \%(0 / 100) \\ \text { add_b + normal } & 4.000 \%(4 / 100) \\ \text { add_b + uniform } & 1.000 \%(1 / 100) \\ \text { add_b + exponential } & 3.000 \%(3 / 100) \\ \text { add_c + normal } & 0.000 \%(0 / 100) \\ \text { add_c + uniform } & 0.000 \%(0 / 100) \\ \text { add_c + exponential } & 0.000 \%(0 / 100) \\ \text { mul_a + normal } & 0.000 \%(0 / 100) \\ \text { mul_a + uniform } & 0.000 \%(0 / 100) \\ \text { mul_a + exponential } & 35.000 \%(35 / 100) \\ \text { mul_b + normal } & 0.000 \%(0 / 100) \\ \text { mul_b + uniform } & 0.000 \%(0 / 100) \\ \text { mul_b + exponential } & 0.000 \%(0 / 100) \\ \text { mul_c + normal } & 6.000 \%(6 / 100) \\ \text { mul_c + uniform } & 4.000 \%(4 / 100) \\ \text { mul_c + exponential } & 28.000 \%(28 / 100) \\ & \end{array}$

com_a + normal

com_a + uniform

com_a + exponential

com_b + normal

com_b + uniform

com_b + exponential

com_c + normal

com_c + uniform

com_c + exponential

$0.000 \%(0 / 100)$

$95.000 \%(95 / 100)$

$0.000 \%(0 / 100)$

$26.000 \%(26 / 100)$

$100.000 \%(100 / 100)$

$3.000 \%(3 / 100)$

$0.000 \%(0 / 100)$

$0.000 \%(0 / 100)$

$0.000 \%(0 / 100)$

\section{Correct predictions averaged for function type:}

$\begin{array}{ll}\text { lin_a } & 0.333 \%(1 / 300) \\ \text { lin_b } & 9.333 \%(28 / 300) \\ \text { lin_c } & 0.000 \%(0 / 300) \\ \text { add_a } & 0.000 \%(0 / 300) \\ \text { add_b } & 2.667 \%(8 / 300) \\ \text { add_c } & 0.000 \%(0 / 300) \\ \text { mul_a } & 11.667 \%(35 / 300) \\ \text { mul_b } & 0.000 \%(0 / 300) \\ \text { mul_c } & 12.667 \%(38 / 300) \\ \text { com_a } & 31.667 \%(95 / 300) \\ \text { com_b } & 43.000 \%(129 / 300) \\ \text { com_c } & 0.000 \%(0 / 300)\end{array}$

\section{Correct predictions averaged for noise type:}

normal: $5.250 \%(63 / 1200)$

uniform: $16.750 \%(201 / 1200)$

exponential: $5.833 \%(70 / 1200)$

Note: Strongly affected by hyperparameter! 


\section{KCDC Results for Synthetic (1000)}

Correct estimates overall: overall: $9.917 \%$ (357 / 3600, bootstrap err. $0.491 \%)$

$\begin{array}{ll}\text { Correct predictions for } & \text { individual setups: } \\ \text { lin_a + normal } & 0.000 \%(0 / 100) \\ \text { lin_a + uniform } & 0.000 \%(0 / 100) \\ \text { lin_a + exponential } & 0.000 \%(0 / 100) \\ \text { lin_b + normal } & 52.000 \%(52 / 100) \\ \text { lin_b + uniform } & 0.000 \%(0 / 100) \\ \text { lin_b + exponential } & 0.000 \%(0 / 100) \\ \text { lin_c + normal } & 0.000 \%(0 / 100) \\ \text { lin_c + uniform } & 0.000 \%(0 / 100) \\ \text { lin_c + exponential } & 0.000 \%(0 / 100) \\ \text { add_a + normal } & 0.000 \%(0 / 100) \\ \text { add_a + uniform } & 0.000 \%(0 / 100) \\ \text { add_a + exponential } & 0.000 \%(0 / 100) \\ \text { add_b + normal } & 0.000 \%(0 / 100) \\ \text { add_b + uniform } & 0.000 \%(0 / 100) \\ \text { add_b + exponential } & 0.000 \%(0 / 100) \\ \text { add_c + normal } & 0.000 \%(0 / 100) \\ \text { add_c + uniform } & 0.000 \%(0 / 100) \\ \text { add_c + exponential } & 0.000 \%(0 / 100) \\ \text { mul_a + normal } & 0.000 \%(0 / 100) \\ \text { mul_a + uniform } & 0.000 \%(0 / 100) \\ \text { mul_a + exponential } & 66.000 \%(66 / 100) \\ \text { mul_b + normal } & 0.000 \%(0 / 100) \\ \text { mul_b + uniform } & 0.000 \%(0 / 100) \\ \text { mul_b + exponential } & 0.000 \%(0 / 100) \\ \text { mul_c + normal } & 0.000 \%(0 / 100) \\ \text { mul_c + uniform } & 0.000 \%(0 / 100) \\ \text { mul_c + exponential } & 38.000 \%(38 / 100)\end{array}$

$\begin{array}{ll}\text { com_a + normal } & 0.000 \%(0 / 100) \\ \text { com_a + uniform } & 100.000 \%(100 / 100) \\ \text { com_a + exponential } & 0.000 \%(0 / 100) \\ \text { com_b + normal } & 1.000 \%(1 / 100) \\ \text { com_b + uniform } & 100.000 \%(100 / 100) \\ \text { com_b + exponential } & 0.000 \%(0 / 100) \\ \text { com_c + normal } & 0.000 \%(0 / 100) \\ \text { com_c + uniform } & 0.000 \%(0 / 100) \\ \text { com_c + exponential } & 0.000 \%(0 / 100)\end{array}$

Correct predictions averaged for function type:

$\begin{array}{ll}\text { lin_a } & 0.000 \%(0 / 300) \\ \text { lin_b } & 17.333 \%(52 / 300) \\ \text { lin_c } & 0.000 \%(0 / 300) \\ \text { add_a } & 0.000 \%(0 / 300) \\ \text { add_b } & 0.000 \%(0 / 300) \\ \text { add_c } & 0.000 \%(0 / 300) \\ \text { mul_a } & 22.000 \%(66 / 300) \\ \text { mul_b } & 0.000 \%(0 / 300) \\ \text { mul_c } & 12.667 \%(38 / 300) \\ \text { com_a } & 33.333 \%(100 / 300) \\ \text { com_b } & 33.667 \%(101 / 300) \\ \text { com_c } & 0.000 \%(0 / 300)\end{array}$

Correct predictions averaged for noise type:

normal: $4.417 \%(53 / 1200)$

uniform: $16.667 \%(200 / 1200)$

exponential: $8.667 \%(104 / 1200)$

Note: Strongly affected by hyperparameter! 


\section{LiNGAM Results for Synthetic (100)}

Correct estimates overall: overall: $1.694 \%$ (61 / 3600, bootstrap err. $0.215 \%)$

$\begin{array}{ll}\text { Correct predictions for } & \text { individual setups: } \\ \text { lin_a + normal } & 34.000 \%(34 / 100) \\ \text { lin_a + uniform } & 7.000 \%(7 / 100) \\ \text { lin_a + exponential } & 10.000 \%(10 / 100) \\ \text { lin_b + normal } & 0.000 \%(0 / 100) \\ \text { lin_b + uniform } & 0.000 \%(0 / 100) \\ \text { lin_b + exponential } & 0.000 \%(0 / 100) \\ \text { lin_c + normal } & 0.000 \%(0 / 100) \\ \text { lin_c + uniform } & 0.000 \%(0 / 100) \\ \text { lin_c + exponential } & 0.000 \%(0 / 100) \\ \text { add_a + normal } & 0.000 \%(0 / 100) \\ \text { add_a + uniform } & 0.000 \%(0 / 100) \\ \text { add_a + exponential } & 0.000 \%(0 / 100) \\ \text { add_b + normal } & 0.000 \%(0 / 100) \\ \text { add_b + uniform } & 0.000 \%(0 / 100) \\ \text { add_b + exponential } & 0.000 \%(0 / 100) \\ \text { add_c + normal } & 0.000 \%(0 / 100) \\ \text { add_c + uniform } & 0.000 \%(0 / 100) \\ \text { add_c + exponential } & 0.000 \%(0 / 100) \\ \text { mul_a + normal } & 0.000 \%(0 / 100) \\ \text { mul_a + uniform } & 0.000 \%(0 / 100) \\ \text { mul_a + exponential } & 0.000 \%(0 / 100) \\ \text { mul_b + normal } & 0.000 \%(0 / 100) \\ \text { mul_b + uniform } & 0.000 \%(0 / 100) \\ \text { mul_b + exponential } & 0.000 \%(0 / 100) \\ \text { mul_c + normal } & 0.000 \%(0 / 100) \\ \text { mul_c + uniform } & 0.000 \%(0 / 100) \\ \text { mul_c + exponential } & 0.000 \%(0 / 100)\end{array}$

com_a + normal

com_a + uniform

$0.000 \%(0 / 100)$

com_a + exponential

$6.000 \%(6 / 100)$

com_b + normal

com_b + uniform

com_b + exponential

com_c + normal

com_c + uniform

com_c + exponential

$1.000 \%$

$3.000 \%(3 / 100)$

$0.000 \%(0 / 100)$

$0.000 \%(0 / 100)$

$0.000 \%(0 / 100)$

$0.000 \%(0 / 100)$

Correct predictions averaged for function type:

$\begin{array}{ll}\text { lin_a } & 17.000 \%(51 / 300) \\ \text { lin_b } & 0.000 \%(0 / 300) \\ \text { lin_c } & 0.000 \%(0 / 300) \\ \text { add_a } & 0.000 \%(0 / 300) \\ \text { add_b } & 0.000 \%(0 / 300) \\ \text { add_c } & 0.000 \%(0 / 300) \\ \text { mul_a } & 0.000 \%(0 / 300) \\ \text { mul_b } & 0.000 \%(0 / 300) \\ \text { mul_c } & 0.000 \%(0 / 300) \\ \text { com_a } & 2.000 \%(6 / 300) \\ \text { com_b } & 1.333 \%(4 / 300) \\ \text { com_c } & 0.000 \%(0 / 300)\end{array}$

Correct predictions averaged for noise type:

normal: $2.917 \%(35 / 1200)$

uniform: $1.333 \%(16 / 1200)$

exponential: $0.833 \%(10 / 1200)$

Note: No data normalization! 


\section{LiNGAM Results for Synthetic (1000)}

Correct estimates overall: overall: $1.667 \%$ (60 / 3600, bootstrap err. $0.213 \%)$

$\begin{array}{ll}\text { Correct predictions for } & \text { individual setups: } \\ \text { lin_a + normal } & 33.000 \%(33 / 100) \\ \text { lin_a + uniform } & 0.000 \%(0 / 100) \\ \text { lin_a + exponential } & 0.000 \%(0 / 100) \\ \text { lin_b + normal } & 0.000 \%(0 / 100) \\ \text { lin_b + uniform } & 0.000 \%(0 / 100) \\ \text { lin_b + exponential } & 0.000 \%(0 / 100) \\ \text { lin_c + normal } & 0.000 \%(0 / 100) \\ \text { lin_c + uniform } & 0.000 \%(0 / 100) \\ \text { lin_c + exponential } & 0.000 \%(0 / 100) \\ \text { add_a + normal } & 0.000 \%(0 / 100) \\ \text { add_a + uniform } & 0.000 \%(0 / 100) \\ \text { add_a + exponential } & 0.000 \%(0 / 100) \\ \text { add_b + normal } & 0.000 \%(0 / 100) \\ \text { add_b + uniform } & 0.000 \%(0 / 100) \\ \text { add_b + exponential } & 0.000 \%(0 / 100) \\ \text { add_c + normal } & 0.000 \%(0 / 100) \\ \text { add_c + uniform } & 0.000 \%(0 / 100) \\ \text { add_c + exponential } & 0.000 \%(0 / 100) \\ \text { mul_a + normal } & 0.000 \%(0 / 100) \\ \text { mul_a + uniform } & 0.000 \%(0 / 100) \\ \text { mul_a + exponential } & 0.000 \%(0 / 100) \\ \text { mul_b + normal } & 0.000 \%(0 / 100) \\ \text { mul_b + uniform } & 0.000 \%(0 / 100) \\ \text { mul_b + exponential } & 0.000 \%(0 / 100) \\ \text { mul_c + normal } & 0.000 \%(0 / 100) \\ \text { mul_c + uniform } & 0.000 \%(0 / 100) \\ \text { mul_c + exponential } & 0.000 \%(0 / 100) \\ & \end{array}$

com_a + normal

com $a+$ uniform

$0.000 \%(0 / 100)$

com_a + exponential

$4.000 \%(4 / 100)$

com_b + normal

com_b + uniform

com_b + exponential

com_c + normal

com_c + uniform

com_c + exponential

$0.000 \%(0 / 100$

$23.000 \%(23 / 100)$

$0.000 \%(0 / 100)$

$0.000 \%(0 / 100)$

$0.000 \%(0 / 100)$

$0.000 \%(0 / 100)$

Correct predictions averaged for function type:

$\begin{array}{ll}\text { lin_a } & 11.000 \%(33 / 300) \\ \text { lin_b } & 0.000 \%(0 / 300) \\ \text { lin_c } & 0.000 \%(0 / 300) \\ \text { add_a } & 0.000 \%(0 / 300) \\ \text { add_b } & 0.000 \%(0 / 300) \\ \text { add_c } & 0.000 \%(0 / 300) \\ \text { mul_a } & 0.000 \%(0 / 300) \\ \text { mul_b } & 0.000 \%(0 / 300) \\ \text { mul_c } & 0.000 \%(0 / 300) \\ \text { com_a } & 1.333 \%(4 / 300) \\ \text { com_b } & 7.667 \%(23 / 300) \\ \text { com_c } & 0.000 \%(0 / 300)\end{array}$

Correct predictions averaged for noise type:

normal: $2.750 \%(33 / 1200)$

uniform: $2.250 \%(27 / 1200)$

exponential: $0.000 \%(0 / 1200)$

Note: No data normalization! 


\section{noTEARS Results for Synthetic (100)}

Correct estimates overall: overall: $86.278 \%$ (3106 / 3600, bootstrap err. $0.578 \%)$

$\begin{array}{ll}\text { Correct predictions for } & \text { individual setups: } \\ \text { lin_a + normal } & 88.000 \%(88 / 100) \\ \text { lin_a + uniform } & 94.000 \%(94 / 100) \\ \text { lin_a + exponential } & 87.000 \%(87 / 100) \\ \text { lin_b + normal } & 78.000 \%(78 / 100) \\ \text { lin_b + uniform } & 58.000 \%(58 / 100) \\ \text { lin_b + exponential } & 86.000 \%(86 / 100) \\ \text { lin_c + normal } & 92.000 \%(92 / 100) \\ \text { lin_c + uniform } & 40.000 \%(40 / 100) \\ \text { lin_c + exponential } & 27.000 \%(27 / 100) \\ \text { add_a + normal } & 100.000 \%(100 / 100) \\ \text { add_a + uniform } & 100.000 \%(100 / 100) \\ \text { add_a + exponential } & 100.000 \%(100 / 100) \\ \text { add_b + normal } & 97.000 \%(97 / 100) \\ \text { add_b + uniform } & 98.000 \%(98 / 100) \\ \text { add_b + exponential } & 97.000 \%(97 / 100) \\ \text { add_c + normal } & 99.000 \%(99 / 100) \\ \text { add_c + uniform } & 100.000 \%(100 / 100) \\ \text { add_c + exponential } & 100.000 \%(100 / 100) \\ \text { mul_a + normal } & 100.000 \%(100 / 100) \\ \text { mul_a + uniform } & 100.000 \%(100 / 100) \\ \text { mul_a + exponential } & 100.000 \%(100 / 100) \\ \text { mul_b + normal } & 100.000 \%(100 / 100) \\ \text { mul_b + uniform } & 100.000 \%(100 / 100) \\ \text { mul_b + exponential } & 94.000 \%(94 / 100) \\ \text { mul_c + normal } & 99.000 \%(99 / 100) \\ \text { mul_c + uniform } & 99.000 \%(99 / 100) \\ \text { mul_c + exponential } & 93.000 \%(93 / 100)\end{array}$

com_a + normal

com_a + uniform

com_a + exponential

com_b + normal

com_b + uniform

com_b + exponential

com_c + normal

com_c + uniform

com $\mathrm{c}+$ exponential
$64.000 \%(64 / 100)$ $12.000 \%(12 / 100)$ $97.000 \%(97 / 100)$ $73.000 \%(73 / 100)$ $50.000 \%(50 / 100)$ $84.000 \%(84 / 100)$ $100.000 \%(100 / 100)$ $100.000 \%(100 / 100)$ $100.000 \%(100 / 100)$

Correct predictions averaged for function type:

$\begin{array}{ll}\text { lin_a } & 89.667 \%(269 / 300) \\ \text { lin_b } & 74.000 \%(222 / 300) \\ \text { lin_c } & 53.000 \%(159 / 300) \\ \text { add_a } & 100.000 \%(300 / 300) \\ \text { add_b } & 97.333 \%(292 / 300) \\ \text { add_c } & 99.667 \%(299 / 300) \\ \text { mul_a } & 100.000 \%(300 / 300) \\ \text { mul_b } & 98.000 \%(294 / 300) \\ \text { mul_c } & 97.000 \%(291 / 300) \\ \text { com_a } & 57.667 \%(173 / 300) \\ \text { com_b } & 69.000 \%(207 / 300) \\ \text { com_c } & 100.000 \%(300 / 300)\end{array}$

Correct predictions averaged for noise type: normal: $90.833 \%(1090 / 1200)$ uniform: $79.250 \%(951 / 1200)$ exponential: $88.750 \%(1065 / 1200)$ 


\section{noTEARS Results for Synthetic (1000)}

Correct estimates overall: overall: $77.139 \%$ (2777 / 3600, bootstrap err. $0.692 \%)$

$\begin{array}{ll}\text { Correct predictions for } & \text { individual setups: } \\ \text { lin_a + normal } & 99.000 \%(99 / 100) \\ \text { lin_a + uniform } & 99.000 \%(99 / 100) \\ \text { lin_a + exponential } & 99.000 \%(99 / 100) \\ \text { lin_b + normal } & 39.000 \%(39 / 100) \\ \text { lin_b + uniform } & 9.000 \%(9 / 100) \\ \text { lin_b + exponential } & 95.000 \%(95 / 100) \\ \text { lin_c + normal } & 91.000 \%(91 / 100) \\ \text { lin_c + uniform } & 7.000 \%(7 / 100) \\ \text { lin_c + exponential } & 0.000 \%(0 / 100) \\ \text { add_a + normal } & 100.000 \%(100 / 100) \\ \text { add_a + uniform } & 100.000 \%(100 / 100) \\ \text { add_a + exponential } & 100.000 \%(100 / 100) \\ \text { add_b + normal } & 97.000 \%(97 / 100) \\ \text { add_b + uniform } & 92.000 \%(92 / 100) \\ \text { add_b + exponential } & 95.000 \%(95 / 100) \\ \text { add_c + normal } & 98.000 \%(98 / 100) \\ \text { add_c + uniform } & 99.000 \%(99 / 100) \\ \text { add_c + exponential } & 98.000 \%(98 / 100) \\ \text { mul_a + normal } & 100.000 \%(100 / 100) \\ \text { mul_a + uniform } & 100.000 \%(100 / 100) \\ \text { mul_a + exponential } & 98.000 \%(98 / 100) \\ \text { mul_b + normal } & 92.000 \%(92 / 100) \\ \text { mul_b + uniform } & 97.000 \%(97 / 100) \\ \text { mul_b + exponential } & 65.000 \%(65 / 100) \\ \text { mul_c + normal } & 97.000 \%(97 / 100) \\ \text { mul_c + uniform } & 94.000 \%(94 / 100) \\ \text { mul_c + exponential } & 70.000 \%(70 / 100)\end{array}$

com_a + normal

com_a + uniform

com_a + exponential

com_b + normal

com_b + uniform

com_c + normal

com_c + uniform

com $\mathrm{c}+$ exponential com_b + exponential

\author{
$44.000 \%(44 / 100)$ \\ $0.000 \%(0 / 100)$ \\ $67.000 \%(67 / 100)$ \\ $45.000 \%(45 / 100)$ \\ $6.000 \%(6 / 100)$ \\ $85.000 \%(85 / 100)$ \\ $100.000 \%(100 / 100)$ \\ $100.000 \%(100 / 100)$ \\ $100.000 \%(100 / 100)$
}

\section{Correct predictions averaged for function type:}

$\begin{array}{ll}\text { lin_a } & 99.000 \%(297 / 300) \\ \text { lin_b } & 47.667 \%(143 / 300) \\ \text { lin_c } & 32.667 \%(98 / 300) \\ \text { add_a } & 100.000 \%(300 / 300) \\ \text { add_b } & 94.667 \%(284 / 300) \\ \text { add_c } & 98.333 \%(295 / 300) \\ \text { mul_a } & 99.333 \%(298 / 300) \\ \text { mul_b } & 84.667 \%(254 / 300) \\ \text { mul_c } & 87.000 \%(261 / 300) \\ \text { com_a } & 37.000 \%(111 / 300) \\ \text { com_b } & 45.333 \%(136 / 300) \\ \text { com_c } & 100.000 \%(300 / 300)\end{array}$

Correct predictions averaged for noise type:

normal: $83.500 \%(1002 / 1200)$

uniform: $66.917 \%(803 / 1200)$

exponential: $81.000 \%(972 / 1200)$ 
Correct estimates overall: overall: $47.417 \%$ (1707 / 3600, bootstrap err. $0.826 \%)$

$\begin{array}{ll}\text { Correct predictions for } & \text { individual setups: } \\ \text { lin_a + normal } & 33.000 \%(33 / 100) \\ \text { lin_a + uniform } & 35.000 \%(35 / 100) \\ \text { lin_a + exponential } & 25.000 \%(25 / 100) \\ \text { lin_b + normal } & 29.000 \%(29 / 100) \\ \text { lin_b + uniform } & 29.000 \%(29 / 100) \\ \text { lin_b + exponential } & 43.000 \%(43 / 100) \\ \text { lin_c + normal } & 91.000 \%(91 / 100) \\ \text { lin_c + uniform } & 100.000 \%(100 / 100) \\ \text { lin_c+ exponential } & 6.000 \%(6 / 100) \\ \text { add_a + normal } & 42.000 \%(42 / 100) \\ \text { add_a + uniform } & 44.000 \%(44 / 100) \\ \text { add_a + exponential } & 57.000 \%(57 / 100) \\ \text { add_b + normal } & 35.000 \%(35 / 100) \\ \text { add_b + uniform } & 63.000 \%(63 / 100) \\ \text { add_b + exponential } & 61.000 \%(61 / 100) \\ \text { add_c + normal } & 29.000 \%(29 / 100) \\ \text { add_c + uniform } & 39.000 \%(39 / 100) \\ \text { add_c + exponential } & 36.000 \%(36 / 100) \\ \text { mul_a + normal } & 74.000 \%(74 / 100) \\ \text { mul_a + uniform } & 86.000 \%(86 / 100) \\ \text { mul_a + exponential } & 48.000 \%(48 / 100) \\ \text { mul_b + normal } & 69.000 \%(69 / 100) \\ \text { mul_b + uniform } & 48.000 \%(48 / 100) \\ \text { mul_b + exponential } & 15.000 \%(15 / 100) \\ \text { mul_c + normal } & 36.000 \%(36 / 100) \\ \text { mul_c + uniform } & 10.000 \%(10 / 100) \\ \text { mul_c + exponential } & 23.000 \%(23 / 100) \\ & \end{array}$

com_a + normal

com_a + uniform

com_a + exponential

com_b + normal

com_b + uniform

com_b + exponential

com_c + normal

com_c + uniform

com_c + exponential

$89.000 \%(89 / 100)$ $90.000 \%(90 / 100)$ $4.000 \%(4 / 100)$ $59.000 \%(59 / 100)$ $55.000 \%(55 / 100)$ $56.000 \%(56 / 100)$ $48.000 \%(48 / 100)$ $56.000 \%(56 / 100)$ $44.000 \%(44 / 100)$

Correct predictions averaged for function type:

$\begin{array}{ll}\text { lin_a } & 31.000 \%(93 / 300) \\ \text { lin_b } & 33.667 \%(101 / 300) \\ \text { lin_c } & 65.667 \%(197 / 300) \\ \text { add_a } & 47.667 \%(143 / 300) \\ \text { add_b } & 53.000 \%(159 / 300) \\ \text { add_c } & 34.667 \%(104 / 300) \\ \text { mul_a } & 69.333 \%(208 / 300) \\ \text { mul_b } & 44.000 \%(132 / 300) \\ \text { mul_c } & 23.000 \%(69 / 300) \\ \text { com_a } & 61.000 \%(183 / 300) \\ \text { com_b } & 56.667 \%(170 / 300) \\ \text { com_c } & 49.333 \%(148 / 300)\end{array}$

Correct predictions averaged for noise type: normal: $52.833 \%(634 / 1200)$ uniform: $54.583 \%(655 / 1200)$ exponential: $34.833 \%(418 / 1200)$ 
Correct estimates overall: overall: $46.361 \%$ (1669 / 3600, bootstrap err. $0.841 \%)$

$\begin{array}{ll}\text { Correct predictions for } & \text { individual setups: } \\ \text { lin_a + normal } & 39.000 \%(39 / 100) \\ \text { lin_a + uniform } & 29.000 \%(29 / 100) \\ \text { lin_a + exponential } & 6.000 \%(6 / 100) \\ \text { lin_b + normal } & 0.000 \%(0 / 100) \\ \text { lin_b + uniform } & 0.000 \%(0 / 100) \\ \text { lin_b + exponential } & 1.000 \%(1 / 100) \\ \text { lin_c + normal } & 50.000 \%(50 / 100) \\ \text { lin_c + uniform } & 92.000 \%(92 / 100) \\ \text { lin_c + exponential } & 0.000 \%(0 / 100) \\ \text { add_a + normal } & 98.000 \%(98 / 100) \\ \text { add_a + uniform } & 100.000 \%(100 / 100) \\ \text { add_a + exponential } & 100.000 \%(100 / 100) \\ \text { add_b + normal } & 88.000 \%(88 / 100) \\ \text { add_b + uniform } & 46.000 \%(46 / 100) \\ \text { add_b + exponential } & 0.000 \%(0 / 100) \\ \text { add_c + normal } & 60.000 \%(60 / 100) \\ \text { add_c + uniform } & 67.000 \%(67 / 100) \\ \text { add_c + exponential } & 63.000 \%(63 / 100) \\ \text { mul_a + normal } & 84.000 \%(84 / 100) \\ \text { mul_a + uniform } & 100.000 \%(100 / 100) \\ \text { mul_a + exponential } & 100.000 \%(100 / 100) \\ \text { mul_b + normal } & 96.000 \%(96 / 100) \\ \text { mul_b + uniform } & 72.000 \%(72 / 100) \\ \text { mul_b + exponential } & 0.000 \%(0 / 100) \\ \text { mul_c + normal } & 45.000 \%(45 / 100) \\ \text { mul_c + uniform } & 0.000 \%(0 / 100) \\ \text { mul_c + exponential } & 0.000 \%(0 / 100) \\ & \end{array}$

com_a + normal

com_a + uniform

com_a + exponential

com_b + normal

com_b + uniform

com_b + exponential

com_c + normal

com_c + uniform

com_c + exponential

$68.000 \%(68 / 100)$

$4.000 \%(4 / 100)$

$0.000 \%(0 / 100)$

$65.000 \%(65 / 100)$

$79.000 \%(79 / 100)$

$50.000 \%(50 / 100)$

$12.000 \%(12 / 100)$

$35.000 \%(35 / 100)$

$20.000 \%(20 / 100)$

Correct predictions averaged for function type:

$\begin{array}{ll}\text { lin_a } & 24.667 \%(74 / 300) \\ \text { lin_b } & 0.333 \%(1 / 300) \\ \text { lin_c } & 47.333 \%(142 / 300) \\ \text { add_a } & 99.333 \%(298 / 300) \\ \text { add_b } & 44.667 \%(134 / 300) \\ \text { add_c } & 63.333 \%(190 / 300) \\ \text { mul_a } & 94.667 \%(284 / 300) \\ \text { mul_b } & 56.000 \%(168 / 300) \\ \text { mul_c } & 15.000 \%(45 / 300) \\ \text { com_a } & 24.000 \%(72 / 300) \\ \text { com_b } & 64.667 \%(194 / 300) \\ \text { com_c } & 22.333 \%(67 / 300)\end{array}$

Correct predictions averaged for noise type:

normal: $58.750 \%(705 / 1200)$

uniform: $52.000 \%(624 / 1200)$

exponential: $28.333 \%(340 / 1200)$ 
Correct estimates overall: $86.944 \%$ (3130 / 3600, bootstrap err. $0.561 \%)$

$\begin{array}{ll}\text { Correct predictions for } & \text { individual setups: } \\ \text { lin_a + normal } & 52.000 \%(52 / 100) \\ \text { lin_a + uniform } & 41.000 \%(41 / 100) \\ \text { lin_a + exponential } & 92.000 \%(92 / 100) \\ \text { lin_b + normal } & 60.000 \%(60 / 100) \\ \text { lin_b + uniform } & 0.000 \%(0 / 100) \\ \text { lin_b + exponential } & 57.000 \%(57 / 100) \\ \text { lin_c + normal } & 99.000 \%(99 / 100) \\ \text { lin_c + uniform } & 6.000 \%(6 / 100) \\ \text { lin_c + exponential } & 100.000 \%(100 / 100) \\ \text { add_a + normal } & 95.000 \%(95 / 100) \\ \text { add_a + uniform } & 90.000 \%(90 / 100) \\ \text { add_a + exponential } & 96.000 \%(96 / 100) \\ \text { add_b + normal } & 100.000 \%(100 / 100) \\ \text { add_b + uniform } & 100.000 \%(100 / 100) \\ \text { add_b + exponential } & 100.000 \%(100 / 100) \\ \text { add_c + normal } & 100.000 \%(100 / 100) \\ \text { add_c + uniform } & 100.000 \%(100 / 100) \\ \text { add_c + exponential } & 100.000 \%(100 / 100) \\ \text { mul_a + normal } & 100.000 \%(100 / 100) \\ \text { mul_a + uniform } & 96.000 \%(96 / 100) \\ \text { mul_a + exponential } & 100.000 \%(100 / 100) \\ \text { mul_b + normal } & 100.000 \%(100 / 100) \\ \text { mul_b + uniform } & 100.000 \%(100 / 100) \\ \text { mul_b + exponential } & 100.000 \%(100 / 100) \\ \text { mul_c + normal } & 99.000 \%(99 / 100) \\ \text { mul_c + uniform } & 99.000 \%(99 / 100) \\ \text { mul_c + exponential } & 100.000 \%(100 / 100) \\ & \end{array}$

com_a + normal

com_a + uniform

com_a + exponential

com_b + normal

com_b + uniform

com_b + exponential

com_c + normal

com_c + uniform

com_c + exponential

$93.000 \%(93 / 100)$

$67.000 \%(67 / 100)$

$100.000 \%(100 / 100)$

$97.000 \%(97 / 100)$

$91.000 \%(91 / 100)$

$100.000 \%(100 / 100)$

$100.000 \%(100 / 100)$

$100.000 \%(100 / 100)$

$100.000 \%(100 / 100)$

Correct predictions averaged for function type:

lin_a $\quad 61.667 \%(185 / 300)$

lin_b $\quad 39.000 \%(117 / 300)$

lin_c $\quad 68.333 \%(205 / 300)$

add_a $\quad 93.667 \%(281 / 300)$

add_b $\quad 100.000 \%(300 / 300)$

add_c $\quad 100.000 \%(300 / 300)$

mul_a $98.667 \%(296 / 300)$

mul_b $\quad 100.000 \%(300 / 300)$

mul_c $\quad 99.333 \%(298 / 300)$

com_a $\quad 86.667 \%(260 / 300)$

com_b $\quad 96.000 \%(288 / 300)$

com_c $\quad 100.000 \%(300 / 300)$

Correct predictions averaged for noise type:

normal: $91.250 \%(1095 / 1200)$

uniform: $74.167 \%(890 / 1200)$

exponential: $95.417 \%(1145 / 1200)$ 
Correct estimates overall: $89.639 \%$ (3227 / 3600, bootstrap err. $0.499 \%)$

$\begin{array}{ll}\text { Correct predictions for individual setups: } \\ \text { lin_a + normal } & 43.000 \%(43 / 100) \\ \text { lin_a + uniform } & 43.000 \%(43 / 100) \\ \text { lin_a + exponential } & 99.000 \%(99 / 100) \\ \text { lin_b + normal } & 60.000 \%(60 / 100) \\ \text { lin_b + uniform } & 0.000 \%(0 / 100) \\ \text { lin_b + exponential } & 86.000 \%(86 / 100) \\ \text { lin_c + normal } & 100.000 \%(100 / 100) \\ \text { lin_c + uniform } & 0.000 \%(0 / 100) \\ \text { lin_c + exponential } & 100.000 \%(100 / 100) \\ \text { add_a + normal } & 100.000 \%(100 / 100) \\ \text { add_a + uniform } & 100.000 \%(100 / 100) \\ \text { add_a + exponential } & 100.000 \%(100 / 100) \\ \text { add_b + normal } & 100.000 \%(100 / 100) \\ \text { add_b + uniform } & 100.000 \%(100 / 100) \\ \text { add_b + exponential } & 100.000 \%(100 / 100) \\ \text { add_c + normal } & 100.000 \%(100 / 100) \\ \text { add_c + uniform } & 100.000 \%(100 / 100) \\ \text { add_c + exponential } & 100.000 \%(100 / 100) \\ \text { mul_a + normal } & 100.000 \%(100 / 100) \\ \text { mul_a + uniform } & 100.000 \%(100 / 100) \\ \text { mul_a + exponential } & 100.000 \%(100 / 100) \\ \text { mul_b + normal } & 100.000 \%(100 / 100) \\ \text { mul_b + uniform } & 100.000 \%(100 / 100) \\ \text { mul_b + exponential } & 100.000 \%(100 / 100) \\ \text { mul_c + normal } & 100.000 \%(100 / 100) \\ \text { mul_c + uniform } & 100.000 \%(100 / 100) \\ \text { mul_c + exponential } & 100.000 \%(100 / 100) \\ & \end{array}$

com_a + normal

com_a + uniform

com_a + exponential

com_b + normal

com_b + uniform

com_b + exponential

com_c + normal

com_c + uniform

com_c + exponential

$100.000 \%(100 / 100)$ $96.000 \%(96 / 100)$ $100.000 \%(100 / 100)$ $100.000 \%(100 / 100)$ $100.000 \%(100 / 100)$ $100.000 \%(100 / 100)$ $100.000 \%(100 / 100)$ $100.000 \%(100 / 100)$ $100.000 \%(100 / 100)$

Correct predictions averaged for function type:

$\begin{array}{ll}\text { lin_a } & 61.667 \%(185 / 300) \\ \text { lin_b } & 48.667 \%(146 / 300) \\ \text { lin_c } & 66.667 \%(200 / 300) \\ \text { add_a } & 100.000 \%(300 / 300) \\ \text { add_b } & 100.000 \%(300 / 300) \\ \text { add_c } & 100.000 \%(300 / 300) \\ \text { mul_a } & 100.000 \%(300 / 300) \\ \text { mul_b } & 100.000 \%(300 / 300) \\ \text { mul_c } & 100.000 \%(300 / 300) \\ \text { com_a } & 98.667 \%(296 / 300) \\ \text { com_b } & 100.000 \%(300 / 300) \\ \text { com_c } & 100.000 \%(300 / 300)\end{array}$

Correct predictions averaged for noise type: normal: $91.917 \%(1103 / 1200)$ uniform: $78.250 \%$ (939 / 1200) exponential: $98.750 \%(1185 / 1200)$ 


\section{Synthetic Results}

\begin{tabular}{lll}
\hline & Synthetic (100) & Synthetic (1000) \\
\hline ANM & $\begin{array}{l}48.361 \%(1741 / 3600) \\
\text { bootstrap err. } 0.812 \%\end{array}$ & $\begin{array}{l}48.139 \%(1733 / 3600) \\
\text { bootstrap err. } 0.844 \%\end{array}$ \\
CAM & $\begin{array}{l}70.389 \%(2534 / 3600) \\
\text { bootstrap err. } 0.748 \%\end{array}$ & $\begin{array}{l}69.306 \%(2495 / 3600) \\
\text { bootstrap err. } 0.769 \%\end{array}$ \\
CCDr & $50.028 \%(1801 / 3600)$ & $47.028 \%(1693 / 3600)$ \\
& bootstrap err. $0.832 \%$ & bootstrap err. $0.806 \%$ \\
CDS & $53.472 \%(1925 / 3600)$ & $51.139 \%(1841 / 3600)$ \\
& bootstrap err. $0.832 \%$ & bootstrap err. $0.817 \%$ \\
IGCI & $93.389 \%(3362 / 3600)$ & $95.306 \%(3431 / 3600)$ \\
& bootstrap err. $0.415 \%$ & bootstrap err. $0.352 \%$ \\
KCDC & $9.278 \%(334 / 3600)$ & $9.917 \%(357 / 3600)$ \\
& bootstrap err. $0.478 \%$ & bootstrap err. $0.491 \%$ \\
LiNGAM & $1.694 \%(61 / 3600)$ & $1.667 \%(60 / 3600)$ \\
& bootstrap err. $0.215 \%$ & bootstrap err. $0.213 \%$ \\
noTEARS & $86.278 \%(3106 / 3600)$ & $77.139 \%(2777 / 3600)$ \\
& bootstrap err. $0.578 \%$ & bootstrap err. $0.692 \%$ \\
RCC & $47.417 \%(1707 / 3600)$ & $46.361 \%(1669 / 3600)$ \\
& bootstrap err. $0.826 \%$ & bootstrap err. $0.841 \%$ \\
RECI & $86.944 \%(3130 / 3600)$ & $89.639 \%(3227 / 3600)$ \\
& bootstrap err. $0.561 \%$ & bootstrap err. $0.499 \%$ \\
\hline
\end{tabular}




\section{Application Data}

Application data allows for obtaining further performance indicators close to real-world problems.

We use data proposed by [Goudet et al., 2018]:

$7 \mathrm{CE}$-Cha: restricted set of continuous variable pairs from the cause effect pair challenge by [Guyon, 2013]

$>$ CE-Gauss: pairs generated by random Gaussian processes with Gaussian mixtures for the cause

7 CE-Multi: pairs generated with linear and polynomial mechanisms

7 CE-Net: pairs generated with a randomly initialized neural network with various distributions of the cause

We use further the well-known Tübingen cause-effect pairs (TCEP) dataset by [Mooij et al., 2016]. 


\begin{tabular}{|c|c|c|c|c|}
\hline & CE-Cha & CE-Gauss & CE-Multi & CE-Net \\
\hline ANM & $\begin{array}{l}67.333 \%(202 / 300) \\
\text { bootstrap err. } 2.626 \%\end{array}$ & $\begin{array}{l}80.000 \%(240 / 300) \\
\text { bootstrap err. } 2.302 \%\end{array}$ & $\begin{array}{l}35.333 \%(106 / 300) \\
\text { bootstrap err. } 2.686 \%\end{array}$ & $\begin{array}{l}76.333 \%(229 / 300) \\
\text { bootstrap err. } 2.344 \%\end{array}$ \\
\hline CAM & $\begin{array}{l}46.667 \%(140 / 300) \\
\text { bootstrap err. } 2.869 \%\end{array}$ & $\begin{array}{l}24.000 \%(72 / 300) \\
\text { bootstrap err. } 2.523 \%\end{array}$ & $\begin{array}{l}34.667 \%(104 / 300) \\
\text { bootstrap err. } 2.664 \%\end{array}$ & $\begin{array}{l}78.333 \%(235 / 300) \\
\text { bootstrap err. } 2.403 \%\end{array}$ \\
\hline CCDr & $\begin{array}{l}47.333 \%(142 / 300) \\
\text { bootstrap err. } 2.778 \%\end{array}$ & $\begin{array}{l}48.667 \%(146 / 300) \\
\text { bootstrap err. } 2.949 \%\end{array}$ & $\begin{array}{l}49.667 \%(149 / 300) \\
\text { bootstrap err. } 2.888 \%\end{array}$ & $\begin{array}{l}53.667 \%(161 / 300) \\
\text { bootstrap err. } 2.747 \%\end{array}$ \\
\hline CDS & $\begin{array}{l}71.000 \%(213 / 300) \\
\text { bootstrap err. } 2.570 \%\end{array}$ & $\begin{array}{l}84.000 \%(252 / 300) \\
\text { bootstrap err. } 2.138 \%\end{array}$ & $\begin{array}{l}43.667 \%(131 / 300) \\
\text { bootstrap err. } 2.850 \%\end{array}$ & $\begin{array}{l}78.333 \%(235 / 300) \\
\text { bootstrap err. } 2.442 \%\end{array}$ \\
\hline $\mathrm{IGCl}$ & $\begin{array}{l}57.333 \%(172 / 300) \\
\text { bootstrap err. } 2.840 \%\end{array}$ & $\begin{array}{l}21.333 \%(64 / 300) \\
\text { bootstrap err. } 2.376 \%\end{array}$ & $\begin{array}{l}68.000 \%(204 / 300) \\
\text { bootstrap err. } 2.743 \%\end{array}$ & $\begin{array}{l}56.333 \%(169 / 300) \\
\text { bootstrap err. } 2.926 \%\end{array}$ \\
\hline KCDC & $\begin{array}{l}49.000 \%(147 / 300) \\
\text { bootstrap err. } 2.929 \%\end{array}$ & $\begin{array}{l}61.667 \%(185 / 300) \\
\text { bootstrap err. } 2.847 \%\end{array}$ & $\begin{array}{l}89.000 \%(267 / 300) \\
\text { bootstrap err. } 1.780 \%\end{array}$ & $\begin{array}{l}60.667 \%(182 / 300) \\
\text { bootstrap err. } 2.730 \%\end{array}$ \\
\hline LiNGAM & $\begin{array}{l}41.667 \%(125 / 300) \\
\text { bootstrap err. } 2.834 \%\end{array}$ & $\begin{array}{l}22.000 \%(66 / 300) \\
\text { bootstrap err. } 2.398 \%\end{array}$ & $\begin{array}{l}30.667 \%(92 / 300) \\
\text { bootstrap err. } 2.601 \%\end{array}$ & $\begin{array}{l}60.667 \%(182 / 300) \\
\text { bootstrap err. } 2.850 \%\end{array}$ \\
\hline noTEARS & $\begin{array}{l}41.000 \%(123 / 300) \\
\text { bootstrap err. } 2.804 \%\end{array}$ & $\begin{array}{l}49.333 \%(148 / 300) \\
\text { bootstrap err. } 2.999 \%\end{array}$ & $\begin{array}{l}39.333 \%(118 / 300) \\
\text { bootstrap err. } 2.890 \%\end{array}$ & $\begin{array}{l}24.333 \%(73 / 300) \\
\text { bootstrap err. } 2.530 \%\end{array}$ \\
\hline $\mathrm{RCC}$ & $\begin{array}{l}46.667 \%(140 / 300) \\
\text { bootstrap err. } 2.843 \%\end{array}$ & $\begin{array}{l}67.667 \%(203 / 300) \\
\text { bootstrap err. } 2.687 \%\end{array}$ & $\begin{array}{l}44.667 \%(134 / 300) \\
\text { bootstrap err. } 2.885 \%\end{array}$ & $\begin{array}{l}73.333 \%(220 / 300) \\
\text { bootstrap err. } 2.578 \%\end{array}$ \\
\hline $\mathrm{RECl}$ & $\begin{array}{l}56.000 \%(168 / 300) \\
\text { bootstrap err. } 2.806 \%\end{array}$ & $\begin{array}{l}64.333 \%(193 / 300) \\
\text { bootstrap err. } 2.771 \%\end{array}$ & $\begin{array}{l}85.333 \%(256 / 300) \\
\text { bootstrap err. } 2.052 \%\end{array}$ & $\begin{array}{l}60.333 \%(181 / 300) \\
\text { bootstrap err. } 2.812 \%\end{array}$ \\
\hline
\end{tabular}




\begin{tabular}{|c|c|c|}
\hline & TCEP v1.0 (plain) & TCEP v1.0 (weighted) \\
\hline ANM & $\begin{array}{l}52.632 \%(50 / 95) \\
\text { bootstrap err. } 5.079 \%\end{array}$ & $\begin{array}{l}52.936 \% \\
\text { bootstrap err. } 6.909 \%\end{array}$ \\
\hline CAM & $\begin{array}{l}60.000 \%(57 / 95) \\
\text { bootstrap err. } 4.911 \%\end{array}$ & $\begin{array}{l}59.238 \% \\
\text { bootstrap err. } 6.597 \%\end{array}$ \\
\hline $\mathrm{CCDr}$ & $\begin{array}{l}73.684 \%(70 / 95) \\
\text { bootstrap err. } 4.549 \%\end{array}$ & $\begin{array}{l}72.142 \% \\
\text { bootstrap err. } 6.152 \%\end{array}$ \\
\hline CDS & $\begin{array}{l}68.421 \%(65 / 95) \\
\text { bootstrap err. } 4.889 \%\end{array}$ & $\begin{array}{l}66.222 \% \\
\text { bootstrap err. } 6.595 \%\end{array}$ \\
\hline IGCI & $\begin{array}{l}34.737 \%(33 / 95) \\
\text { bootstrap err. } 5.101 \%\end{array}$ & $\begin{array}{l}33.569 \% \\
\text { bootstrap err. } 6.515 \%\end{array}$ \\
\hline $\mathrm{KCDC}$ & $\begin{array}{l}51.579 \%(49 / 95) \\
\text { bootstrap err. } 5.197 \%\end{array}$ & $\begin{array}{l}54.816 \% \\
\text { bootstrap err. } 6.756 \%\end{array}$ \\
\hline LiNGAM & $\begin{array}{l}50.526 \%(48 / 95) \\
\text { bootstrap err. } 5.057 \%\end{array}$ & $\begin{array}{l}37.732 \% \\
\text { bootstrap err. } 6.244 \%\end{array}$ \\
\hline noTEARS & $\begin{array}{l}48.421 \%(46 / 95) \\
\text { bootstrap err. } 5.104 \%\end{array}$ & $\begin{array}{l}50.831 \% \\
\text { bootstrap err. } 6.545 \%\end{array}$ \\
\hline $\mathrm{RCC}$ & $\begin{array}{l}38.947 \%(37 / 95) \\
\text { bootstrap err. } 4.978 \%\end{array}$ & $\begin{array}{l}42.413 \% \\
\text { bootstrap err. } 6.633 \%\end{array}$ \\
\hline $\mathrm{RECl}$ & $\begin{array}{l}62.105 \%(59 / 95) \\
\text { bootstrap err. } 4.958 \%\end{array}$ & $\begin{array}{l}69.223 \% \\
\text { bootstrap err. } 5.946 \%\end{array}$ \\
\hline
\end{tabular}




\section{Summary}

$>$ This is an intermediate state of an ongoing investigation.

$>$ Methods are realized by the code provided by authors, the CausalDiscoveryToolbox by [Kalainathan and Goudet, 2019], or are own implementations.

$>$ There is no hyperparameter optimization, dataset normalization, or fine-tuning of the methods involved (we always use default settings).

$>$ We plan to utilize further datasets such as more cause effect challenge pairs by [Guyon, 2013]

7 We also have many more methods to evaluate (results already partially available): [Zhang and Hyvärinen, 2008, Mooij et al., 2010, de Almeida, 2013, Sgouritsa et al., 2015, Bontempi and Flauder, 2015, Hernandez-Lobato et al., 2016, Fonollosa, 2016, Budhathoki and Vreeken, 2017, Lopez-Paz et al., 2017, Ramsey et al., 2017, Goudet et al., 2018, Kalainathan et al., 2018, Chen et al., 2019, Zhu and Chen, 2019, Gnecco et al., 2019, Cai et al., 2019, Goldfarb and Evans, 2019, Monti et al., 2019, de Almeida, 2019, Samothrakis et al., 2019, Minnaert, 2019, Strobl and Visweswaran, 2019]

$>$ Please tell us if we missed some interesting methods / datasets: christoph.kaeding@dlr.de 


\section{References I}

Aragam, B. and Zhou, Q. (2015).

Concave penalized estimation of sparse gaussian bayesian networks.

Journal of Machine Learning Research.

Blöbaum, P., Janzing, D., Washio, T., Shimizu, S., and Schölkopf, B. (2018).

Cause-effect inference by comparing regression errors.

In International Conference on Artificial Intelligence and Statistics.

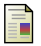

Bontempi, G. and Flauder, M. (2015).

From dependency to causality: a machine learning approach.

The Journal of Machine Learning Research (JMLR).

Budhathoki, K. and Vreeken, J. (2017).

Causal inference by stochastic complexity.

arXiv preprint arXiv:1702.06776.

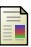

Bühlmann, P., Peters, J., Ernest, J., et al. (2014).

Cam: Causal additive models, high-dimensional order search and penalized regression.

The Annals of Statistics.

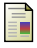

Cai, R., Qiao, J., Zhang, K., Zhang, Z., and Hao, Z. (2019).

Causal discovery with cascade nonlinear additive noise models.

arXiv preprint arXiv: 1905.09442.

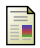

Chen, Z., Zhu, S., Liu, Y., and Tse, T. (2019).

Causal discovery by kernel intrinsic invariance measure.

arXiv preprint arXiv:1909.00513. 


\section{References II}

Daniusis, P., Janzing, D., Mooij, J., Zscheischler, J., Steudel, B., Zhang, K., and Schölkopf, B. (2012).

Inferring deterministic causal relations.

arXiv preprint arXiv:1203.3475.

De Almeida, D. M. (2013).

Automated feature engineering applied to causality.

In NIPS Workshop on Causality.

De Almeida, D. M. (2019).

Pattern-based causal feature extraction.

In Cause Effect Pairs in Machine Learning.

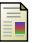

Fonollosa, J. A. (2016).

Conditional distribution variability measures for causality detection.

arXiv preprint arXiv:1601.06680.

Enecco, N., Meinshausen, N., Peters, J., and Engelke, S. (2019).

Causal discovery in heavy-tailed models.

arXiv preprint arXiv:1908.05097.

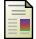

Goldfarb, D. and Evans, S. (2019).

Causal inference via conditional kolmogorov complexity using mdl binning.

arXiv preprint arXiv:1911.00332.

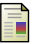

Goudet, O., Kalainathan, D., Caillou, P., Guyon, I., Lopez-Paz, D., and Sebag, M. (2018).

Learning functional causal models with generative neural networks.

In Explainable and Interpretable Models in Computer Vision and Machine Learning. 


\section{References III}

Guyon, I. (2013).

Chalearn cause effect pairs challenge.

http://www.causality.inf.ethz.ch/cause-effect.php.

Hernandez-Lobato, D., Morales-Mombiela, P., Lopez-Paz, D., and Suarez, A. (2016).

Non-linear causal inference using gaussianity measures.

The Journal of Machine Learning Research (JMLR).

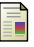

Hoyer, P. O., Janzing, D., Mooij, J. M., Peters, J., and Schölkopf, B. (2009).

Nonlinear causal discovery with additive noise models.

In Advances in Neural Information Processing Systems.

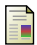

Kalainathan, D. and Goudet, O. (2019).

Causal discovery toolbox: Uncover causal relationships in python.

arXiv preprint arXiv:1903.02278.

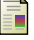

Kalainathan, D., Goudet, O., Guyon, I., Lopez-Paz, D., and Sebag, M. (2018).

Sam: Structural agnostic model, causal discovery and penalized adversarial learning. arXiv preprint arXiv:1803.04929.

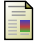

Lopez-Paz, D., Muandet, K., Schölkopf, B., and Tolstikhin, I. (2015).

Towards a learning theory of cause-effect inference.

In International Conference on Machine Learning.

Lopez-Paz, D., Nishihara, R., Chintala, S., Scholkopf, B., and Bottou, L. (2017).

Discovering causal signals in images.

In IEEE Conference on Computer Vision and Pattern Recognition. 


\section{References IV}

Minnaert, B. (2019).

Feature importance in causal inference for numerical and categorical variables.

In Cause Effect Pairs in Machine Learning.

Mitrovic, J., Sejdinovic, D., and Teh, Y. W. (2018).

Causal inference via kernel deviance measures.

In Advances in Neural Information Processing Systems.

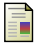

Monti, R. P., Zhang, K., and Hyvarinen, A. (2019).

Causal discovery with general non-linear relationships using non-linear ica.

arXiv preprint arXiv: 1904.09096.

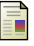

Mooij, J. M., Peters, J., Janzing, D., Zscheischler, J., and Schölkopf, B. (2016).

Distinguishing cause from effect using observational data: methods and benchmarks.

The Journal of Machine Learning Research.

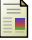

Mooij, J. M., Stegle, O., Janzing, D., Zhang, K., and Schölkopf, B. (2010).

Probabilistic latent variable models for distinguishing between cause and effect.

In Advances in Neural Information Processing Systems.

Ramsey, J., Glymour, M., Sanchez-Romero, R., and Glymour, C. (2017).

A million variables and more: the fast greedy equivalence search algorithm for learning high-dimensional graphical causal models, with an application to functional magnetic resonance images.

International journal of data science and analytics. 


\section{References V}

Samothrakis, S., Perez, D., and Lucas, S. (2019).

Training gradient boosting machines using curve-fitting and information-theoretic features for causal direction detection.

In Cause Effect Pairs in Machine Learning.

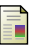

Sgouritsa, E., Janzing, D., Hennig, P., and Schölkopf, B. (2015).

Inference of cause and effect with unsupervised inverse regression.

In Artificial intelligence and statistics.

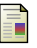

Shimizu, S., Hoyer, P. O., Hyvärinen, A., and Kerminen, A. (2006).

A linear non-gaussian acyclic model for causal discovery.

Journal of Machine Learning Research.

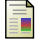

Strobl, E. V. and Visweswaran, S. (2019).

Markov blanket ranking using kernel-based conditional dependence measures.

In Cause Effect Pairs in Machine Learning.

Zhang, K. and Hyvärinen, A. (2008).

Distinguishing causes from effects using nonlinear acyclic causal models.

In International Conference on Causality.

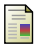

Zheng, X., Aragam, B., Ravikumar, P. K., and Xing, E. P. (2018).

Dags with no tears: Continuous optimization for structure learning.

In Advances in Neural Information Processing Systems.

Zhu, S. and Chen, Z. (2019).

Causal discovery with reinforcement learning.

arXiv preprint arXiv:1906.04477. 